\title{
Effects of collagen fiber addition on the combustion and thermal stability of natural rubber
}

\author{
Weixing $\mathrm{Xu}^{1}$, Xintao $\mathrm{Wu}^{2}$, Qilin Wen ${ }^{1}$, Shuangyang $\mathrm{Li}^{1}$, Yongjiao Song ${ }^{3}$ and Bi Shi ${ }^{1,4^{*}}$
}

\begin{abstract}
Collagen fiber (CF) and silane coupling agent-modified collagen fiber (MCF) were used as flame retardant filler for natural rubber (NR) modification. The combustion phenomena and properties of composites blended with different dosages of CF or MCF were compared to elucidate the flame retardant mechanism of the composites. The flame retardancy of NR can be enhanced effectively by increasing nitrogen content (the nitrogen content of CF is about 18\%), creating air pockets, and structuring the flame retardant network in the composites. MCF failed to structure a flame retardant network in the composite, indicating that its modification effects of MCF are weaker than those of CF. When CF dosage was $30 \mathrm{wt} \%$, the composite can achieve the best flame retardancy, with limited oxygen index of $29.4 \%$ and without smoke and dripping during burning. This study demonstrated a new method for the flame retardant modification of NR.
\end{abstract}

\section{Introduction}

Natural rubber (NR) is an important chemical materials in the modern industry. It can be used as a "commodity polymer", and as an "engineering elastomer" [1]. NR has some unique properties in applications, such as excellent toughness and elasticity, and good alkali resistance. Given these advantages, NR, one of the earliest natural polymers used by mankind, still attracts great attention in material science. However, the inherently high flammability of NR limits its application [1-4].

In general, the flammability of polymer materials can be decreased in two ways. One way is through chemical modification using reagents or monomers containing fire retardant elements [5-7]. Chlorine- or brominecontaining monomers can enhance the flame resistance of NR effectively $[8,9]$. However, addition of halogens triggers the release of toxic gases during combustion

\footnotetext{
* Correspondence: shibi@scu.edu.cn

${ }^{1}$ National Engineering Research Center of Clean Technology in Leather

Industry, Sichuan University, Chengdu 610065, China

${ }^{4}$ College of Chemistry and Materials Science, Sichuan Normal University,

Chengdu 610068, China

Full list of author information is available at the end of the article
}

$[10,11]$. Another way is to introduce a flame-retardant additive (FRA) [2, 4, 12-18]. Many FRAs have been developed to increase the flame retardancy of polymer material. Previous researchers have successfully improved the flame retardancy of NR by using proper FRAs, such as montmorillonite, aluminium hydroxides, and melamine-formaldehyde resin $[2,4,19]$. Most of these works focused on making NR resistant to ignition. The high flammability of NR is reflected in two aspects: NR is easy to ignition; flame spreads quickly across the material following the dripping. Thus, understanding the flame propagation in NR is important.

Flame propagation of polymers during burning can be affected by two methods of flame retardant modification corresponding to two types of carbon residues formed by FRAs in composites. One type is the dense carbon layer covered on the composite surface, which improves the flame retardancy of composites by cutting off air contact (blocking effects) [20-23]. The other type is the continuous porous carbon layer, which improves the flame retardancy of composite by blocking heat propagation (intumescent effects) [15, 24-27]. However, little is known about the blocking effects applied on the flame 
retardant modification of NR. The FRAs used in the flame retardant modification of NR with intumescent effects can be divided into two groups: hyperbranched molecule [3, 4, 28, 29] and microcapsule [2, 30-32]. However, the synthesis cost of these two FRAs is relatively high, which seriously affects their practicability.

Our previous study proved that collagen fiber (CF) has some advantages in preparing efficacious flame retardant filler [33]. In addition, the multilevel structure [34] of CF is similar to the structure of hyperbranched molecule, which is well-designed to achieve a better modification effect. This structure may contribute to the formation of a porous carbon layer that blocks the energy transmission of a combustion flame [35]. As such, CF is expected to be processed into a type of FRA for NR. Moreover, CF is a renewable biomass resource [35-38]. Our previous study also proved that blending with CF does not impair the mechanical properties of NR [39]. Thus, the practicability of CF-based FRA can be guaranteed.

In the present study, we used CF to construct a flame retardant network in NR. We hoped this method could enhance the flame retardancy of NR by blocking the dripping. To achieve this goal, we prepared a series of CF/NR composites. The limited oxygen indexes (LOIs) of these composites were tested. The flame retardant mechanism of NR modified by CF was studied by morphologic characterization and thermogravimetric analysis (TGA).

\section{Experimental}

\subsection{Materials}

Wet-blue splits with an average thickness of $1.6 \mathrm{~mm}$ were obtained from a local tannery in China. Surfactant (FG-B) was commercial grade and supplied by Sichuan Dowell Science \& Technology Inc. (Sichuan, China). Formic acid $(\mathrm{HCOOH})$, acetic acid $\left(\mathrm{CH}_{3} \mathrm{COOH}\right)$, aluminum sulfate octadecahydrate $\left(\mathrm{Al}_{2}\left(\mathrm{SO}_{4}\right)_{3} \cdot 18 \mathrm{H}_{2} \mathrm{O}\right)$, magnesium oxide (MgO), sulfur ( $\mathrm{S}$ ) and isopropyl alcohol were all analytical pure and purchased from Kelong Chemical Reagent Corporation (Sichuan, China). Natural rubber was commercial grade and supplied by Sinopec Group (China). Silane coupling agent (KH-550) was commercial grade and supplied by Nanjing Xiangqian Chemical Co., LTD (Jiangsu, China).

\subsection{Preparation of collagen fiber powder}

Wet-blue splits were washed and wrung, and then processed as in Table 1 in a drum $(\varnothing 30 \mathrm{~cm})$ commonly used in leather processing. After drying, splits were smashed by an Ultra Centrifugal Mill (ZM 200, Retsch, Germany) to obtain collagen fiber powder. The sieve size is 0.08 $\mathrm{mm}$ with trapezoid holes.

\subsection{Preparation of modified collagen fiber powder}

Silane coupling agent, isopropyl alcohol and water were mixed in the ratio of 1:90:10 $(\mathrm{w} / \mathrm{w} / \mathrm{w})$ to get the modification solution. Ten grams collagen fiber powder was impregnated in $60 \mathrm{~g}$ modification solution for $3 \mathrm{~h}$ at $40{ }^{\circ} \mathrm{C}$. During this progress, $\mathrm{pH}$ was checked every 10 min and kept at $6.0 \sim 6.5$ by adding acetic acid. After filtration, collagen fiber powder was heated at $105^{\circ} \mathrm{C}$ for 6 h. After washing and re-smashing, modified collagen fiber (MCF) powder was obtained.

\subsection{Preparation of the CF/NR and the MCF/NR composites} CF or MCF was mixed with NR by a torque rheometer (RM-200C, Harp, China) at $150^{\circ} \mathrm{C}$ for $20 \mathrm{~min} .5 \mathrm{wt} \%$ sulfur (based on the weight of NR) was added as sulfurizing reagent. Then the $\mathrm{CF} / \mathrm{NR}$ and $\mathrm{MCF} / \mathrm{NR}$ composite were prepared by a vulcanizing press (QLB-25 T, Junyu, China) at $150^{\circ} \mathrm{C}$ with a pressure of $1.5 \mathrm{MPa}$ for $15 \mathrm{~min}$.

Table 1 Pretreatment processes of wet-blue split

\begin{tabular}{|c|c|c|c|c|c|}
\hline Step & Material & $\operatorname{Dosage}^{a}(\%)$ & Temperature $\left({ }^{\circ} \mathrm{C}\right)$ & Time (min) & $\mathrm{pH}$ \\
\hline \multirow[t]{2}{*}{ Rewetting } & water & 400 & 35 & 40 & \\
\hline & Surfactant (FG-B) & 0.4 & & & \\
\hline Washing & water & 400 & 35 & 10 & \\
\hline \multirow[t]{4}{*}{ Retanning } & water & 200 & 35 & & \\
\hline & $\mathrm{HCOOH}(1: 10)^{b}$ & $0.2 \times n$ & & $10 \times n+30$ & 3.0 \\
\hline & $\mathrm{Al}_{2}\left(\mathrm{SO}_{4}\right)_{3} \cdot 18 \mathrm{H}_{2} \mathrm{O}$ & 13 & & 60 & \\
\hline & $\mathrm{Al}_{2}\left(\mathrm{SO}_{4}\right)_{3} \cdot 18 \mathrm{H}_{2} \mathrm{O}$ & 13 & & 120 & \\
\hline \multicolumn{6}{|c|}{ Run 50 min per hour for 5 times } \\
\hline Basification & $\mathrm{MgO}$ & $0.1 \times n$ & 25 & $10 \times n+30$ & $\mathrm{pH}=4.0 \sim 4.2$ \\
\hline Washing & water & $100 \times 3$ & 25 & $15 \times 3$ & \\
\hline
\end{tabular}

a: All the quantities were based on the weight of wet-blue splits

${ }^{b}$ : Formic acid was diluted ten times before addition 

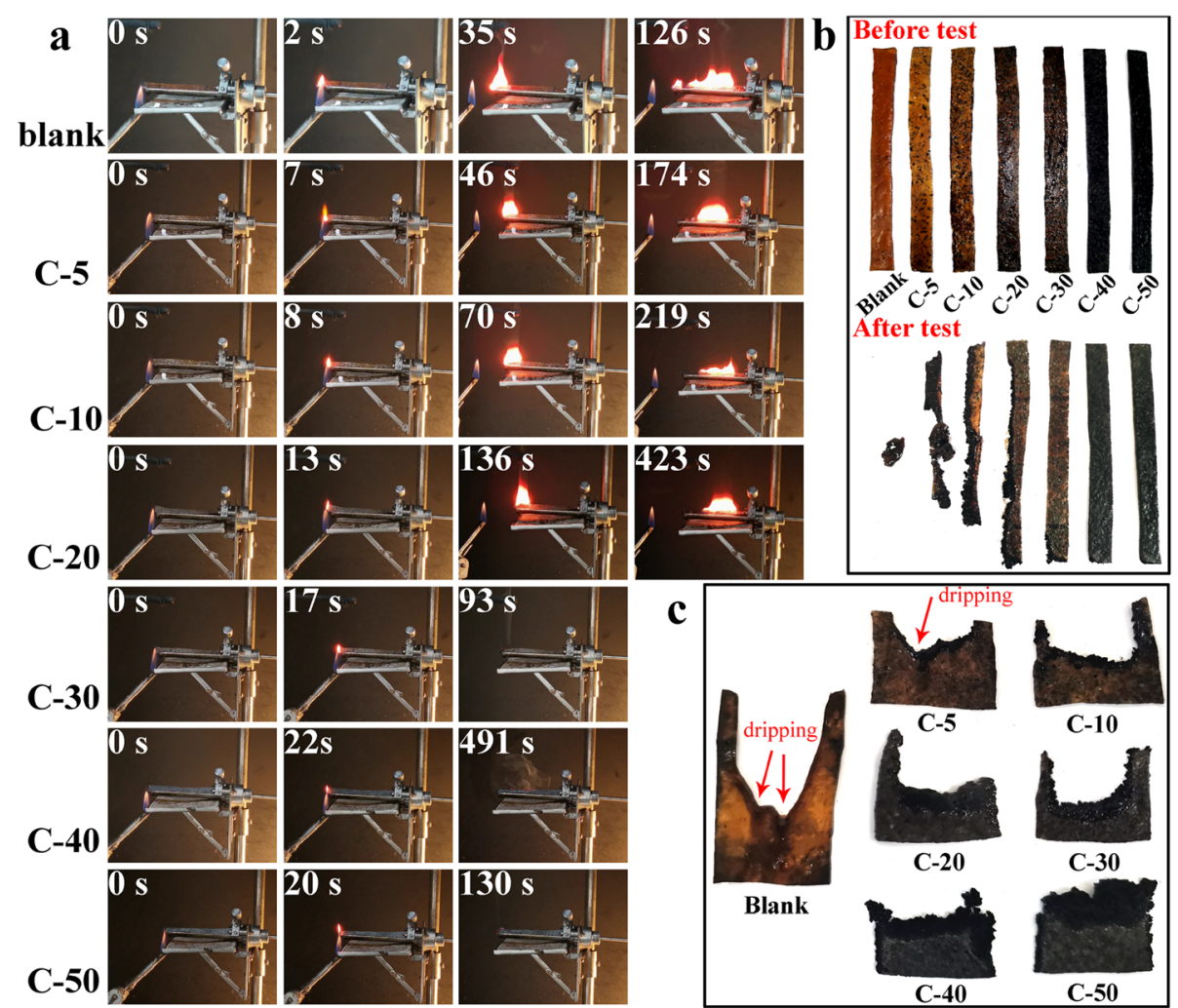

Fig. 1 a snapshots of NR and the CF/NR composites with different CF dosage during the UL94 horizontal burning test. b photos of NR and the CF/NR composites with different CF dosage before and after the UL94 horizontal burning test. c photos of NR and the CF/NR composites with different CF dosage after the LOI test

\subsection{Characterizations}

The morphology of CF and MCF was observed by a stereo microscope (SM, DFC550, Leica, Germany). The morphology of the CF/NR and the MCF/NR composite was observed by a scanning electron microscopy (SEM, SU3500, JEOL, Japan) with an accelerating voltage of $15.0 \mathrm{kV}$. The combustion behavior of NR and the composites was determined using a horizontal and vertical combustion tester (CZF-5CD, Jiangning, China) according to UL 94. The samples for horizontal and vertical combustion test were cut to $130 \mathrm{~mm}$ long, $13 \mathrm{~mm}$ wide and less than $3 \mathrm{~mm}$ thick. The LOI of NR and the composites was determined using an oxygen index meter (JF-3, Jiangning, China) according to ASTM D2863. The samples for LOI test were cut to $140 \mathrm{~mm}$ long, $52 \mathrm{~mm}$ wide and less than $5 \mathrm{~mm}$ thick. The thermal stabilities of CF, MCF, NR and the composites were carried out on a thermogravimetric analyzer (TGA, TGA 8000, PerkinElmer, America) at a heating rate of $10{ }^{\circ} \mathrm{C} / \mathrm{min}$ under air atmosphere. The thermal conductivities of NR and the composites were determined using a thermal constant analyzer (TPS 2500S, Hot Disk, Sweden) according to ISO 22007-2:2015. The nitrogen contents of the $\mathrm{CF} / \mathrm{NR}$ and the $\mathrm{MCF} / \mathrm{NR}$ composites

Table 2 The LOI and combustion phenomena of NR and the CF/NR composites

\begin{tabular}{lllllll}
\hline Sample & CF dosage $(\mathbf{w t} \%)$ & LOI $(\%)$ & Smoke & Dripping & Flying Spark & horizontal burning rate $(\mathbf{m m} / \mathbf{m i n})$ \\
\hline NR & 0 & $17.9 \pm 0.4$ & $(+)$ & $(+)$ & $(-)$ & $49.45 \pm 3.44$ \\
C-5 & 5 & $19.2 \pm 0.4$ & $(+)$ & $(+)$ & $(-)$ & $35.16 \pm 2.84$ \\
C-10 & 10 & $21.9 \pm 0.4$ & $(-)$ & $( \pm)$ & $(-)$ & $30.20 \pm 1.34$ \\
C-20 & 20 & $25.6 \pm 0.6$ & $(-)$ & $(-)$ & $(-)$ & $15.68 \pm 0.94$ \\
C-30 & 30 & $29.4 \pm 0.3$ & $(-)$ & $(-)$ & $(-)$ & self-extinguishing \\
C-40 & 40 & $27.2 \pm 0.6$ & $(-)$ & $(-)$ & $(+)$ & self-extinguishing \\
C-50 & 50 & $25.6 \pm 0.7$ & $(-)$ & $(-)$ & $(+)$ & self-extinguishing \\
\hline
\end{tabular}

$(+)$ : positive; (-): negative; $( \pm)$ : sometimes positive, sometimes negative 

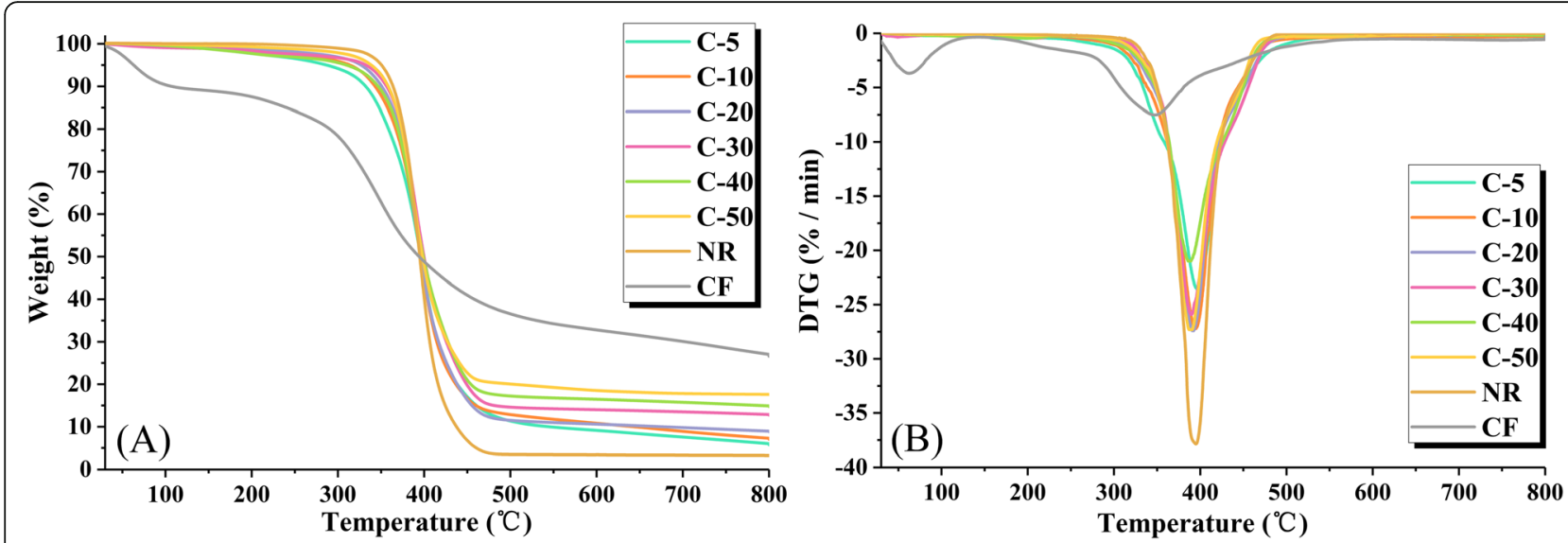

Fig. 2 The TG (a) and DTG (b) curves of NR, CF and CF/NR composites

were detected by a total organic carbon and protein-nitrogen analyzer (Primacs ${ }^{\text {SNC-100 }}$, SKALAR, Netherlands).

\section{Results and discussion}

3.1 Effect of CF dosage on the flammability of CF/NR composites

The flame retardancy of the different NR and CF/NR composites with different CF dosage was estimated by UL94 horizontal/vertical burning test and LOI test (Fig. 1 and Table 2). The flammability of NR obviously reduced with the addition of CF. Figure 1a shows that the time to ignition of the CF/NR composites was prolonged with the addition of CF. In addition, the horizontal burning rate decreased rapidly. When the dosage of CF was higher than $10 \mathrm{wt} \%$, burning smoke can be suppressed. When the CF dosage was $30 \mathrm{wt} \%$, self-extinguishing phenomenon was observed. However, all these samples failed to reach UL94-V2 level (supporting 1). Figure 1b shows the NR and $\mathrm{CF} / \mathrm{NR}$ composites before and after the horizontal burning test. When the CF dosage exceeded $10 \mathrm{wt} \%$, combustion occurred only in the upper layer of the sample. In addition, the CF/NR composites did not contract violently due to combustion. Figure 1c illustrates the NR and CF/ NR composites after the LOI test. When the CF dosage exceeded $10 \mathrm{wt} \%$, dripping can be suppressed significantly. These results proved that CF is a feasible filler for the flame retardant modification of NR.

LOI and the combustion phenomena missed by snapshots are also listed in Table 2. The LOI of the composites initially increased then decreased with increasing CF dosage. However, flying sparks appeared when the CF dosage exceeded $40 \mathrm{wt} \%$. This phenomenon has two possible reasons. First, the active groups (e.g. $-\mathrm{COOH}$,

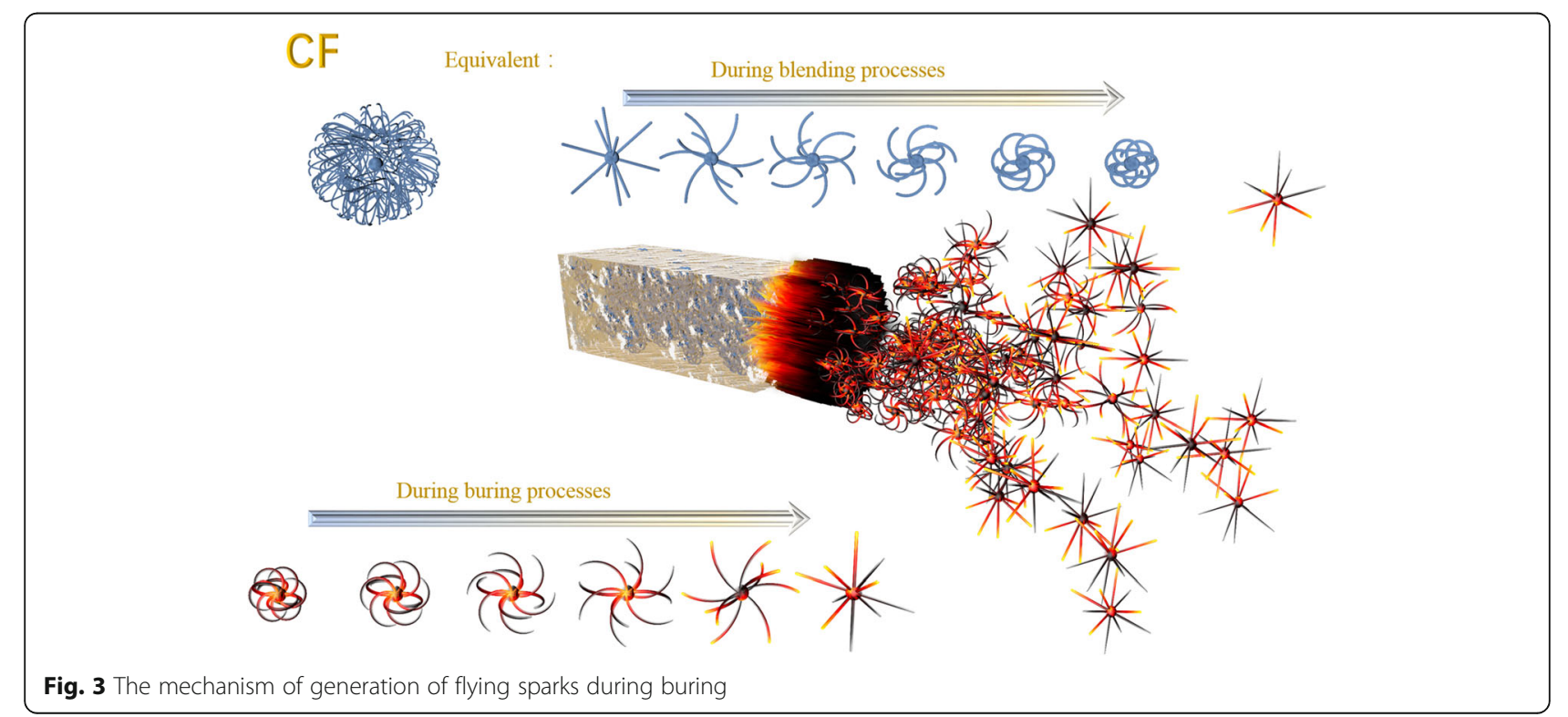




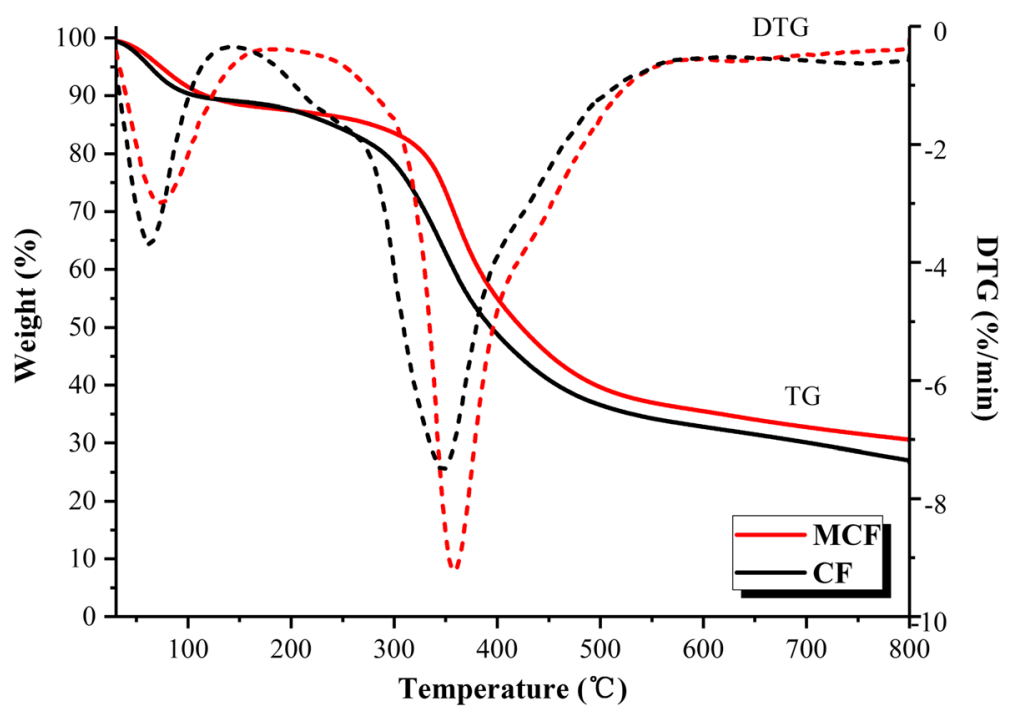

Fig. 4 The TG and DTG curves of CF and MCF

$-\mathrm{NH}_{2}$ and - $\mathrm{OH}$ ) on the collagen molecule may decompose during combustion [40], leading to the release of gases, such as $\mathrm{CO}_{2}$ and $\mathrm{H}_{2} \mathrm{O}$. These gases can blow some residues. Second, a large amount of energy is stored in the composites during mixing and hot-pressing. When $\mathrm{NR}$, as the continuous phase, melts and burns, the energy stored in CF is released. Both reasons explain why the ignited CF scatters in all directions. With the loss of $\mathrm{CF}$, the CF/NR composite becomes easier to burn, while flying sparks can ignite other parts of the sample. When $50 \mathrm{wt} \% \mathrm{CF}$ was added in the composite, this phenomenon became more obvious and LOI was further decreased. The composite with $30 \mathrm{wt} \% \mathrm{CF}$ (C-30) presented the best flame retardancy, where the highest LOI was achieved, and smoke, dripping, and flying spark were suppressed.

The thermal decomposition behaviors of NR, CF, and $\mathrm{CF} / \mathrm{NR}$ composites with different CF dosages were characterized by TGA, as shown in Fig. 2. Except water evaporation $\left(0 \sim 160^{\circ} \mathrm{C}\right)$, only one region of decomposition appeared in the temperature range of $160 \sim 800^{\circ} \mathrm{C}$ for all these samples [41, 42]. The onset decomposition temperature $\left(\mathrm{T}_{\text {onset }}\right)$ and char yield at $800^{\circ} \mathrm{C}$ increased with the increase in $\mathrm{CF}$ dosage. Although the temperature corresponding maximum decomposition rate $\left(\mathrm{T}_{\max }\right)$ of $\mathrm{CF}$ was lower than that of NR, no significant decrease in the $T_{\max }$ of $C F / N R$ composites was found. These phenomena indicate that the addition of CF enhanced the thermal stability of NR. The change regularity of the thermal decomposition characteristics of the CF/NR composites was inconsistent with the LOI test results. Because the thermal decomposition reaction in TGA was not as violent as burning. CF did not burn and scatter rapidly during heating in TGA. Thus, the modification result was not affected.

The labile groups on the collagen molecule decomposed into gases during TGA. No unexpected mass change was observed. The flying sparks observed in the LOI tests were caused by the dramatic release of energy. $\mathrm{CF}$ has a good compressibility. When the movement of $\mathrm{CF}$ is no longer restricted, the tight CFs become loose immediately, whereas the lithe CFs are blown away by

Table 3 The LOI and combustion phenomena of the MCF/NR composites

\begin{tabular}{|c|c|c|c|c|c|}
\hline Sample & MCF dosage (wt\%) & LOI (\%) & Smoke & Dripping & Flying Spark \\
\hline NR & 0 & $17.9 \pm 0.4$ & $(+)$ & $(+)$ & $(-)$ \\
\hline$C-30$ & 30 (CF) & $29.4 \pm 0.3$ & $(-)$ & $(-)$ & $(-)$ \\
\hline MC-5 & 5 & $20.4 \pm 0.3$ & $(-)$ & $(+)$ & $(-)$ \\
\hline$M C-10$ & 10 & $22.3 \pm 0.4$ & $(-)$ & $(+)$ & $(-)$ \\
\hline MC-20 & 20 & $23.2 \pm 0.9$ & $(-)$ & $(+)$ & $(-)$ \\
\hline MC-30 & 30 & $24.5 \pm 1.4$ & $(-)$ & $(+)$ & $(-)$ \\
\hline MC-40 & 40 & $25.1 \pm 0.8$ & $(-)$ & $(+)$ & $(-)$ \\
\hline MC-50 & 50 & $25.7 \pm 0.8$ & $(-)$ & $(+)$ & $(-)$ \\
\hline
\end{tabular}

(+): positive, (-): negative 


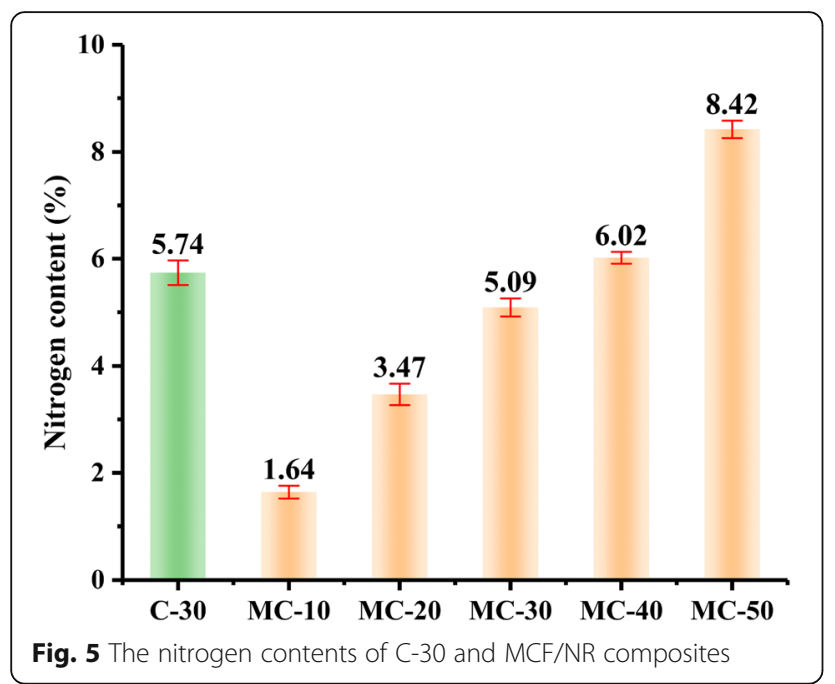

the air easily. This phenomenon explains the flying sparks in the LOI test when the CF dosage was more than $30 \mathrm{wt} \%$. A schematic of the generation of flying sparks during burning is shown in Fig. 3.

\subsection{Effect of MCF dosage on the flammability of MCF/NR composites}

The results above prove that although the addition of $\mathrm{CF}$ can enhance the flame retardancy of NR, the energy stored in the composite can affect the modification results. Given that a high pressure is needed to force NR to become a continuous phase (supporting 2 ), a practical solution is to change the interfacial force between NR and CF. A silane coupling agent was used to improve the interaction between $\mathrm{CF}$ and NR to eliminate the negative influence. Moreover, the introduction of silicon is considered beneficial to the improvement of flame retardancy [43-45]. The TGA curves of $C F$ and MCF are shown in Fig. 4. The $T_{\text {on- }}$ set, $\mathrm{T}_{\max }$, and char yield at $800{ }^{\circ} \mathrm{C}$ of MCF were obviously higher than those of CF. LOI tests showed that the LOIs of MCF and CF were $68.1 \%$ and $60.7 \%$, respectively. These results prove that MCF has a better flame retardancy than $\mathrm{CF}$, implying that MCF has the potential to modify the flame retardancy of NR more effectively than CF.

The LOI test results of the MCF/NR composites with different MCF dosages are shown in Table 3. The LOI obviously increased with increasing MCF Amount. No smoke and flying sparks were found during the test. However, compared with C-30 in Table 2, the MCF/NR composites did not achieve a higher LOI and better flame retardancy. In fact, dripping always occurred during the LOI test of the MCF/NR composites. Flame and energy can travel quickly with the droplets.

The TGA test results (supporting 3) showed that the $\mathrm{T}_{\text {onset, }}, \mathrm{T}_{\max }$ and char yield at $800^{\circ} \mathrm{C}$ of MC-30 were all higher than those of $\mathrm{C}-30$, indicating that the thermal stability of MC-30 was better than that of C-30. These results were inconsistent with the LOI test results possibly because dripping did not occur in the TGA test.

Given that the flame retardancy and thermal stability of MCF were all better than those of CF, the lower LOI of MCF/NR should be caused by the structural change during modification and mixing. After all, LOI is a more important indicator for flame retardant material than char yard. The use of a silane coupling agent to improve the interaction between CF and NR is not a feasible plan to enhance enhancing flame retardant effects in the CF/ NR composite.

\subsection{Flame retardant mechanism of NR modified with CF}

For most flame retardant modifications of polymers, increasing the nitrogen content of the blended system is an effective method [46-48]. In the present study, the addition of CF or MCF can increase the nitrogen content of the blended system. However, it is not a decisive factor. The nitrogen contents of the CF/NR (C-30) and MCF/NR composites are shown in Fig. 5. Even the nitrogen content of MC-50 was much higher than that of C-30, and the LOI of the former was still lower than that of the latter.

The special structure of CF may play an important role in the modification. Figure 6 illustrates the SM photos of $\mathrm{CF}$ and MCF. Obviously, these two fibers have different structures. CF is much fluffier than MCF. Similar
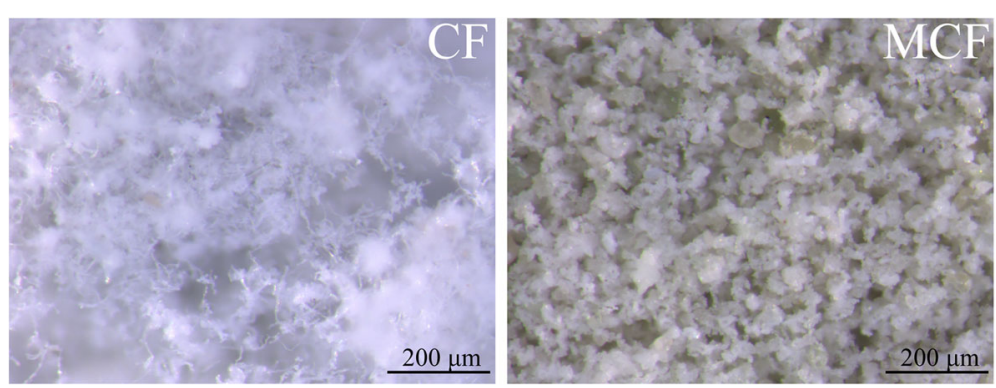

Fig. 6 The SM photos of CF and MCF 


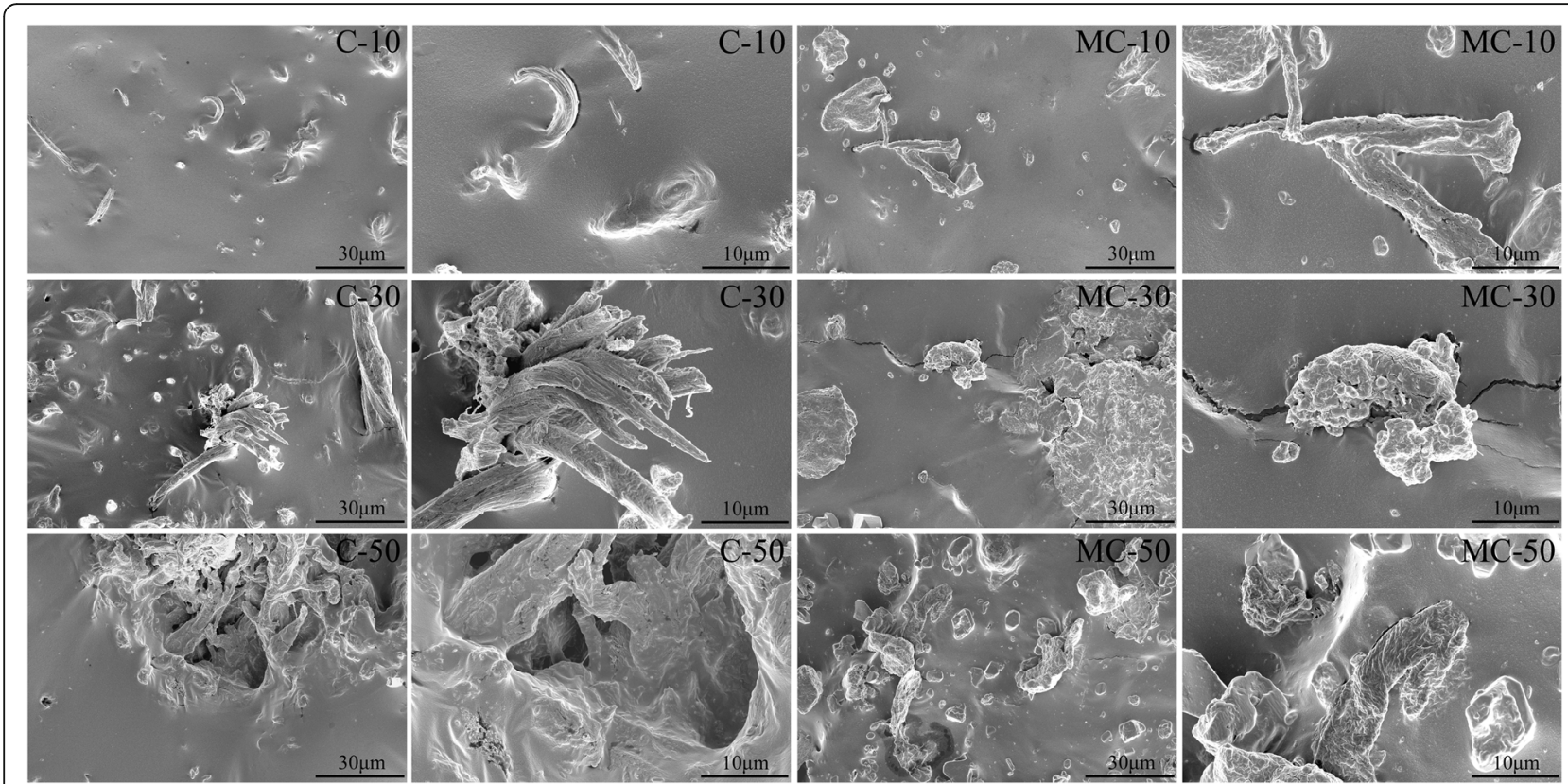

Fig. 7 The SEM photos of different CF/NR composite and MCF/NR composites

phenomena can be also found in the SEM photos of the composites in Fig. 7. The multilevel structure of CF can still be observed in the CF/NR composites. Meanwhile the MCF added in the composite was coated with a dense film, which covers the open-framework structure of CF. Because of this dense structure, a network structure is hard to build, even when the dosage of MCF is $50 \mathrm{wt} \%$. Hence, a semi-interpenetrating network (semi-IPN) of CF is important for the flame retardancy modification of NR. This network can block the spread of flame, and has a great anti-dripping effect [49-52]. The action mechanism and effects of $\mathrm{CF}$ and MCF in

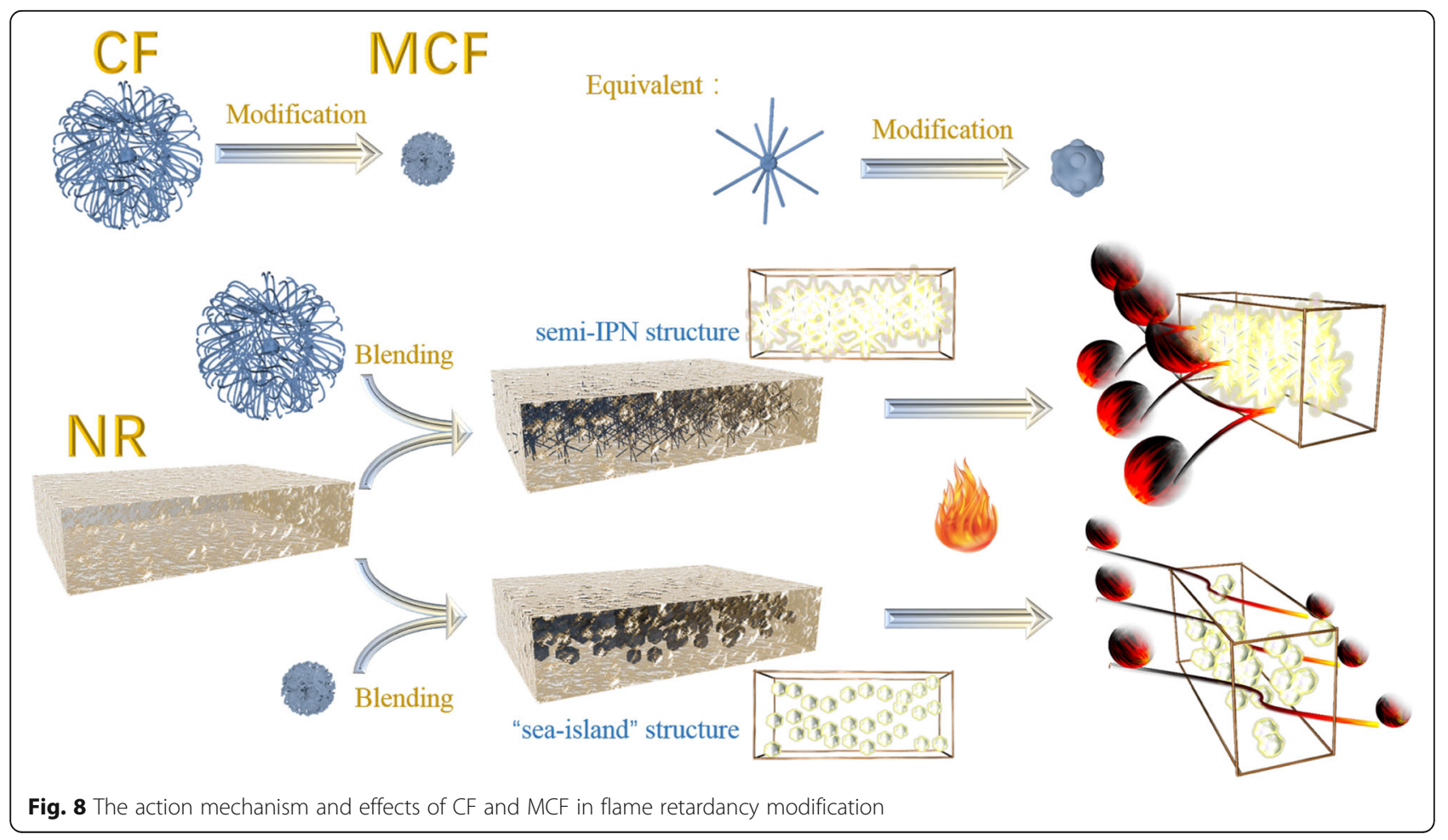


Table 4 The thermal conductivity of NR, C-30 and MC-30

\begin{tabular}{llll}
\hline Sample & NR & C-30 & MC-30 \\
\hline Thermal conductivity $(\mathrm{W} / \mathrm{mK})$ & 0.1733 & 0.1457 & 0.1629 \\
\hline
\end{tabular}

flame retardancy modification of NR are shown in Fig. 8. The fluffy CF can entangle with each other and tends to be semi-interpenetrating in the blend system. While, the MCF can only form the "sea-island" structure in the blend system, which may fail to block the flame propagation effectively.

Table 4 shows the thermal conductivities of NR, C-30 and MC-30. Results showed that the thermal conductivity of C-30 was lower than those of NR and $\mathrm{MC}-30$, indicating that the heat flow propagation in C-30 was more difficult than those in NR and $\mathrm{MC}-30$. A fully dispersed structure of CF can form a certain amount of air pockets in the composite to block heat propagation. By contrast, the unsatisfactory modification result of MCF is caused by the fact that these air pockets are filled up by the silane coupling agent.

Combining with all the above results, the flame retardant mechanism of NR modified with CF has three aspects. First, the nitrogen content of the composite increased. Second, the network structure containing CF formed firebreaks in the composite, which helped impede flame and dripping. Third, the multilevel structure of CF created air pockets in the composite to block heat propagation.

\section{Conclusion}

The flame retardancy of NR can be improved by blending with CF. The addition of CF suppressed smoke and dripping during burning. A flame retardant network and enough air pockets must be structured to ensure the modification effect. As such, CF should be remain fluffy and polyporous. With 30 wt\% dosage of CF, the LOI of modified NR was as high as $29.4 \%$. This composite also had satisfactory $\mathrm{T}_{\text {onset }}$ and char yield at $800{ }^{\circ} \mathrm{C}$. With these properties, the usage of NR can be extended to highly demanding applications.

The practicability of this flame-retardancy modification method should be further investigated. The problem of flying sparks should be solved to improve the flame retardancy of NR. We speculate another compound may be needed to replace the silane coupling agent to enhance compatibility between NR and CF. The improvement of the blending system should also be considered. All these works are undertaken.

\section{Supplementary information}

Supplementary information accompanies this paper at https://doi.org/10. 1186/s42825-020-00040-1.

Additional file 1 Supporting 1: Figure S1 snapshots of C-30 during UL94 vertical burning test. Figure $\mathbf{S 1}$ shows the $\mathrm{C}-30$ during vertical burning test. Results show that even with the highest LOI, C-30 still failed to reach the UL94-V2 level.

Additional file 2 Supporting 2. Figure S2 The SM photos of the CF/ NR composite. Figure $\mathbf{S 2}$ shows the SM photos of the CF/NR composite, when the input quantity in the torque rheometer is only $2 / 3$ of normal samples. NR is covered by CF, and the whole structure is discontinuous.

Additional file $\mathbf{3}$ Supporting 3. Figure $\mathbf{S} 3$ The TGA curves of $\mathrm{C}-30$ and MC-30. Figure S3 displays TGA curves of C-30 and MC-30. The Tonset $\mathrm{T}_{\text {max }}$ and char yield at $800^{\circ} \mathrm{C}$ of $\mathrm{MC}-30$ are all higher than those of $\mathrm{C}-30$, as shown in Table $\mathrm{S} 1$.

Additional file 4 Table S1 The results of TGA of C-30 and MC-30.

\section{Acknowledgements}

The authors gratefully acknowledge Prof. Hui Wang from Analytical \& Testing Center of Sichuan University and Ms. Chenxu Tian from the College of Polymer Science and Engineering of Sichuan University for their help in measurement and characterization.

\section{Authors' contributions}

Weixing Xu: conception of the work, preparation of MCF/NR composite, morphological observation of composite, analysis and interpretation of data, drafting the manuscript. Xintao Wu: characterization of the LOI of materials. Qilin Wen: characterization of the thermostability of materials. Shuangyang Li: Preparation of modified collagen fiber powder. Yongjiao Song: Preparation of CF/NR composite. Bi Shi: conception of the work, substantively revision of the manuscript. All authors have read and approved the submitted version (and any substantially modified version that involves the author's contribution to the study). All authors have agreed both to be personally accountable for the author's own contributions and to ensure that questions related to the accuracy or integrity of any part of the work, even ones in which the author was not personally involved, are appropriately investigated, resolved, and the resolution documented in the literature.

\section{Funding}

The financial support of this study was from the National Natural Science Foundation of China Youth Science Foundation (21808145).

\section{Availability of data and materials}

The datasets used and analyzed during the current study are available from the corresponding author on reasonable request.

Ethics approval and consent to participate Not applicable.

\section{Consent for publication}

Not applicable.

\section{Competing interests}

The authors declare that they have no competing interests.

\section{Author details}

${ }^{1}$ National Engineering Research Center of Clean Technology in Leather Industry, Sichuan University, Chengdu 610065, China. ${ }^{2}$ The Key Laboratory of Leather Chemistry and Engineering of Ministry of Education, Sichuan University, Chengdu 610065, China. ${ }^{3}$ Department of Biomass Science and Engineering, Sichuan University, Chengdu 610065, China. ${ }^{4}$ College of Chemistry and Materials Science, Sichuan Normal University, Chengdu 610068, China. 
Received: 14 May 2020 Accepted: 2 October 2020

Published online: 15 December 2020

\section{References}

1. Wang J, Chen Y. Microencapsulation of intumescent flame-retardant agent: application to flame-retardant natural rubber composite. J Appl Polym Sci. 2007;104(3):1828-38

2. Wang N, Mi L, Wu Y, et al. Double-layered co-microencapsulated ammonium polyphosphate and mesoporous MCM-41 in intumescent flame-retardant natural rubber composites. J Therm Anal Calorim. 2014; 115(2):1173-81.

3. Zhao Y, Wang JC. Preparation of novel hyper-branched flame-retardant polymer and its application into natural rubber systems. J Appl Polym Sci. 2013;128(4):2385-94.

4. Zhang C, Wang J. Natural rubber / dendrimer modified montmorillonite nanocomposites: mechanical and flame-retardant properties. Materials. 2017. https://doi.org/10.3390/ma11010041

5. Wang $X$, Xing W, Feng $X$, et al. Functionalization of graphene with grafted polyphosphamide for flame retardant epoxy composites: synthesis, flammability and mechanism. Polym Chem-HK. 2014;5(4):1145-54.

6. Cheema HA, El-Shafei A, Hauser PJ. Conferring flame retardancy on cotton using novel halogen-free flame retardant bifunctional monomers: synthesis, characterizations and applications. Carbohyd Polym. 2013;92(1):885-93.

7. Guo Z, Wang C, Li J, et al. Micro-intumescent flame retardant polyamide 6 based on cyclic phosphate grafting phenol formaldehyde. Polym Advan Technol. 2016;27(7):955-63.

8. Tan KL, Chen XS, Yan BS, et al. Application of chlorinated waste rubber as a flame retardant of low density polyethylene. J Appl Polym Sci. 2012;123(6): 3495-502.

9. Sutker BJ, Ti GS, Khuddus MA. Brominated fire retardant composition: U.S. Patent 4,728,463. 1988-3-1.

10. Rakotomalala M, Wagner S, Döring M. Recent developments in halogen free flame retardants for epoxy resins for electrical and electronic applications. Materials. 2010;3(8):4300-27.

11. Thirumal M, Singha NK, Khastgir D, et al. Halogen-free flame-retardant rigid polyurethane foams: effect of alumina trihydrate and triphenylphosphate on the properties of polyurethane foams. J Appl Polym Sci. 2010;116(4):2260-8.

12. Karak N, Maiti S. Antimony polymers. III. Flame retardant behavior of chloroprene and natural rubber vulcanizates with antimony polymer. J Appl Polym Sci. 2015;68(6):927-35.

13. Kashiwagi $\mathrm{T}$, Shields JR, Harris JRH, et al. Flame retardant mechanism of a polymer clay nanocomposite. The Fourteenth Annual BCC Conference on Flame Retardancy. 2017.

14. Gu J, Liang C, Zhao X, et al. Highly thermally conductive flame-retardant epoxy nanocomposites with reduced ignitability and excellent electrical conductivities. Compos Sci Technol. 2017;139:83-9.

15. Wang C, Wu Y, Li Y, et al. Flame retardant rigid polyurethane foam with a phosphorus nitrogen single intumescent flame retardant. Polym Advan Technol. 2018;29(1):668-76.

16. Huo S, Yang S, Wang J, et al. A liquid phosphorus-containing imidazole derivative as flame-retardant curing agent for epoxy resin with enhanced thermal latency, mechanical, and flame-retardant performances. J Hazard Mater. 2019;386:121984.

17. Cheng J, Wang J, Yang S, et al. Benzimidazolyl-substituted cyclotriphosphazene derivative as latent flame-retardant curing agent for one-component epoxy resin system with excellent comprehensive performance. Compos Part B-Eng. 2019;177:107440.

18. Simonetti $\mathrm{P}$, Nazir $\mathrm{R}$, Gooneie $\mathrm{A}$, et al. Michael addition in reactive extrusion: a facile sustainable route to developing phosphorus based flame retardant materials. Compos Part B-Eng. 2019;178:107470.

19. Habibah AIHD, Ruhida AR. The effects of halogen-free flame retardant additives in Epoxidized Natural Rubber (ENR). Adv Mater Rev. 2015;1107: $131-6$.

20. Huo S, Wang J, Yang S, et al. Flame-retardant performance and mechanism of epoxy thermosets modified with a novel reactive flame retardant containing phosphorus, nitrogen, and sulfur. Polym Advan Technol. 2018; 29(1):497-506

21. Liu H, Zhang B, Han J. Flame retardancy and smoke suppression properties of flexible polyurethane foams containing an aluminum phosphate microcapsule. RSC Adv. 2017;7(56):35320-9.
22. Guan Q, Yuan L, Wu S, et al. Enhanced thermal and dielectric properties of hybrid organic/inorganic shell microcapsule/thermosetting resin nanocomposites. Polym Int. 2017;66(12):1940-8.

23. Xu M, Liu H, Ma K, et al. New strategy towards flame retardancy through design, synthesis, characterization, and fire performance of a chain extender in polyamide 6 composites. Polym Ehg Sci. 2018. https://doi.org/10.1002/ pen.25030.

24. Chen S, Li X, Li Y, et al. Intumescent flame-retardant and self-healing superhydrophobic coatings on cotton fabric. ACS Nano. 2015;9(4):4070-6.

25. Bocz K, Krain T, Marosi G, et al. Effect of particle size of additives on the flammability and mechanical properties of intumescent flame retarded polypropylene compounds. Int J Polym Sci. 2015. https://doi.org/10.1155/ 2015/493710

26. Chen $X$, Liu L, Jiao C, et al. Influence of ferrite yellow on combustion and smoke suppression properties in intumescent flame-retardant epoxy composites. High Perform Polym. 2015;27(4):412-25.

27. Gavgani JN, Adelnia H, Sadeghi GM, et al. Intumescent flame retardant polyurethane/starch composites: thermal, mechanical, and rheological properties. J Appl Polym Sci. 2014;131(23):41158.

28. Wang J, Guo Y. Hyperbranched intumescent flame-retardant agent: application to natural rubber composites. J Appl Polym Sci. 2011;122(5): 3474-82.

29. Wang N, Zhang M, Kang P, et al. Synergistic effect of graphene oxide and mesoporous structure on flame retardancy of nature rubber/IFR composites. Materials. 2018. https://doi.org/10.3390/ma11061005.

30. Wang $N, X u G, W u Y$, et al. The influence of expandable graphite on double-layered microcapsules in intumescent flame-retardant natural rubber composites. J Therm Anal Calori. 2016;123(2):1239-51.

31. Wang $N, M i L$, Wu $Y$, et al. Enhanced flame retardancy of natural rubber composite with addition of microencapsulated ammonium polyphosphate and MCM-41 fillers. Fire Safety J. 2013. https://doi.org/10.1016/j.firesaf.2013. 09.008

32. Wang J, Chen Y. Effect of microencapsulation and $4 \mathrm{~A}$ zeolite on the properties of intumescent flame-retardant natural rubber composites. J Fire Sci. 2008;26(2):153-71.

33. Wen $\mathrm{Q}, \mathrm{Wu}$ X, Xu W, et al. Effects of dispersion and fixation of collagen fiber network on its flame retardancy. Polym Degrad Stabil. 2020;175:109122.

34. Deng D, Liao $X$, Shi $B$, et al. Synthesis of porous carbon fibers from collagen fiber. Chemsuschem. 2008;1(4):298-301.

35. Bourbigot S, Duquesne S. Fire retardant polymers: recent developments and opportunities. J Mater Chem. 2007;17(22):2283-300.

36. Wei $X$, Zhang $R$, Zhang W, et al. High-efficiency adsorption of tetracycline by the prepared waste collagen fiber-derived porous biochar. RSC Adv. 2019;9(67):39355-66

37. Liu X, Tang R, He Q, et al. Fe (III)-loaded collagen fiber as a heterogeneous catalyst for the photo-assisted decomposition of malachite green. J Hazard Mater. 2010;174(1):687-93.

38. Niu J, Shao R, Liu M, et al. Collagen-derived porous carbons: porous carbons derived from collage-nenriched biomass: tailored design, synthesis, and application in electrochemical energy storage and conversion. Adv Funct Mater. 2019. https://doi.org/10.1002/adfm.201905095.

39. Li S, Wang Y, Xu W, et al. Natural rubber-based elastomer reinforced by chemically modified multiscale leather collagen fibers with excellent toughness. ACS Sustain Chem Eng. 2020. https://doi.org/10.1021/ acssuschemeng.9b07078.

40. Su W, Ma X, Sun $X$, et al. Physicochemical characterisation and thermal decomposition of synthetic collagen based nanocomposites in comparison with natural bone. Adv Appl Ceram. 2016;115(1):29-35.

41. Liu J, Brown EM, Uknalis J, et al. Thermal stability and degradation kinetics of vegetable-tanned collagen fiber with in-situ precipitated calcium carbonate. J Am Leather Chem As. 2018;113(11):358-70.

42. Liu J, Luo L, Hu YD, et al. Kinetics and mechanism of thermal degradation of vegetable-tanned leather fiber. J Leather Sci Eng. 2019;1:9.

43. Ebdon JR, Hunt BJ, Jones MS, et al. Chemical modification of polymers to improve flame retardancy-II. The influence of silicon-containing groups. Polym Degrad Stabil. 1996:54(2-3):395-400.

44. Carosio F, Laufer G, Alongi J, et al. Layer-by-layer assembly of silicabased flame retardant thin film on PET fabric. Polym Degrad Stabil. 2011;96(5):745-50

45. Feng $Y$, He $C$, Wen $Y$, et al. Improving thermal and flame retardant properties of epoxy resin by functionalized graphene containing 
phosphorous, nitrogen and silicon elements. Compos Part A-Appls. 2017; 103:74-83.

46. Gao F, Tong L, Fang Z. Effect of a novel phosphorous-nitrogen containing intumescent flame retardant on the fire retardancy and the thermal behaviour of poly (butylene terephthalate). Polym Degrad Stabil. 2006;91(6): 1295-9.

47. Costes L, Laoutid F, Aguedo M, et al. Phosphorus and nitrogen derivatization as efficient route for improvement of lignin flame retardant action in PLA. Eur Polym J. 2016;84:652-67.

48. Xu W, Yu C, Zhao X, et al. Melamine formaldehyde/polyvinyl alcohol composite fiber: structures and properties controlled by reaction-induced phase separation. J Appl Polym Sci. 2016;133(4):42918.

49. Yang $H$, Guan $Y$, Ye L, et al. Synergistic effect of nanoscale carbon black and ammonium polyphosphate on improving thermal stability and flame retardancy of polypropylene: a reactive network for strengthening carbon layer. Compos Part B-Eng. 2019;174:107038.

50. Zhuo $D$, Wang $R$, Wu $L$, et al. Flame retardancy effects of graphene nanoplatelet / carbon nanotube hybrid membranes on carbon fiber reinforced epoxy composites. J Nanomater. 2013;2013:2745-56.

51. Cao X, Lu K, Li Y. Isolated protective char layers by nanoclay network: significantly improved flame retardancy and mechanical performance of TPV/MH composites by small amount of nanoclay. Ind Eng Chem Res. 2015; 54(27):6912-21.

52. Habibi S, Saket M. Effect of clay network formation on flame retardancy of polyethylene terephthalate / montmorillonite nanocomposites. J Appl Chem Res. 2017;11(4):74-85.

\section{Publisher's Note}

Springer Nature remains neutral with regard to jurisdictional claims in published maps and institutional affiliations.

\section{Submit your manuscript to a SpringerOpen ${ }^{\circ}$ journal and benefit from:}

- Convenient online submission

- Rigorous peer review

- Open access: articles freely available online

- High visibility within the field

- Retaining the copyright to your article

Submit your next manuscript at $\boldsymbol{\nabla}$ springeropen.com 